\title{
Análisis de supervivencia de cáncer de mama localmente avanzado tratado con quimioterapia neoadyuvante
}

\author{
- Pedro Luis Ramos Guette, María Athenas Ramos Escalante, Diana Silva, Mario Gonzáles, Mauricio García \\ Oncológica Oncocare (Bogotá, D.C.)
}

Introducción: el cáncer de mama localmente avanzado tiene un pronóstico adverso y requiere tratamiento con quimioterapia neoadyuvante. El propósito de este estudio es evaluar la supervivencia global y libre de enfermedad en mujeres con cáncer de mama localmente avanzado tratadas con quimioterapia neoadyuvante.

Materiales y métodos: es un estudio analítico de tipo cohorte retrospectiva durante el período enero de 2013-mayo de 2016. Se incluyeron todas las pacientes mayores de 18 años con cáncer de mama estadios IIIA, IIIB y IIIC. Se construyeron curvas de supervivencia con el método de Kaplan-Meier. Se compararon con el método de rangos logarítmicos y análisis de regresión de rangos proporcionales por Cox.

Resultados: se identificaron 379 pacientes con una edad promedio de 54,7 años. El 59,6\% eran posmenopaúsicas. El 68,7\% tiene receptores de estrógenos positivos. El 18,4\% eran HER2 positivos. El tamaño tumoral promedio es $57 \mathrm{~mm}$. El 48,30\% está en estadio IIIB. El 82,85\% tiene ganglios positivos. Tipo histológico ductal $88,65 \%$ y grado II $51,19 \%$. La quimioterapia AC y ACT fue del $84 \%$. Las respuestas completas fueron de un $15,24 \%$. Las recaídas del $24,27 \%$ y la mortalidad del $22,43 \%$. La mediana de seguimiento es de 88 meses. La supervivencia global y libre de enfermedad se ven disminuida en el compromiso ganglionar masivo HR 6,01 (IC95\% 3,20-11,26) y 6,74 (IC95\% 3,26-12,56). Las respuestas patológicas completas aumentan la supervivencia libre de enfermedad HR 0,43 (IC95\% 0,21-0,90), pero no la global HR 0,52 (IC95\% 0,25-1,09).

Conclusiones: la supervivencia global y libre de enfermedad se ve reducida cuando hay un compromiso ganglionar masivo; hay incremento en la supervivencia libre de enfermedad en las respuestas completas. 